\title{
Post-antibiotic effect of orbifloxacin against Escherichia coli and Pseudomonas aeruginosa isolates from dogs
}

\author{
Kazuki Harada*, Takae Shimizu, Yasushi Kataoka and Toshio Takahashi
}

\begin{abstract}
Orbifloxacin is a fluoroquinolone drug used widely in companion animal medicine. In this study, we firstly determined post-antibiotic effects (PAEs) and post-antibiotic sub-minimum inhibitory concentrations (MIC) effects (PA-SMEs) of orbifloxacin for two strains each of Escherichia coli and Pseudomonas aeruginosa from dogs, and these parameters were compared with those of enrofloxacin. At twice the MIC, the PAEs of orbifloxacin ranged from -0.28-0.93 h (mean, 0.29 h) for E. coli and -0.18-1.18 h (mean, $0.37 \mathrm{~h}$ ) for $P$. aeruginosa. These parameters were not significantly different for $E$. coli and shorter for $P$. aeruginosa, compared to enrofloxacin $(P<0.05)$. Continued exposure to $0.1,0.2$, and 0.3 the MIC of orbifloxacin resulted in average PA-SMEs of 0.55, 1.11, and $2.03 \mathrm{~h}$, respectively, for E. coli, and 1.04, 1.40, and 2.47 h, respectively, for P. aeruginosa. These PA-SMEs, which had no significant differences with those of enrofloxacin, were significantly longer than the corresponding PAEs $(P<0.05)$. These results suggest that the PA-SME of orbifloxacin for E. coli and P. aeruginosa can be meaningfully prolonged by increase of sub-MICs.
\end{abstract}

\section{Findings}

Orbifloxacin is a fluoroquinolone developed for use in companion animal medicine. This antimicrobial agent exhibits bactericidal activity against numerous gramnegative and gram-positive bacteria. In canine practice, orbifloxacin is indicated for the treatment of various infections, including urinary, skin, and otitis infections, and is available in many countries including Japan.

Pharmacodynamic variables such as the post-antibiotic effect (PAE) and post-antibiotic sub-minimum inhibitory concentration effect (PA-SME) have increasingly become the focus of investigations designed to determine optimal dosage regimens for antimicrobial agents. The PAE is defined as the length of time that bacterial growth is suppressed following brief exposure to an antibiotic [1]. PAE has been investigated for several veterinary fluoroquinolones, such as enrofloxacin, marbofloxacin, and difloxacin [2-5], but not for orbifloxacin. On the other hand, PA-SME is defined as the time interval that includes the PAE plus the additional time during which

\footnotetext{
* Correspondence: k-harada@nvlu.ac.jp

Laboratory of Veterinary Microbiology, Nippon Veterinary and Life Science University, 1-7-1, Kyonan-cho, Musashino, Tokyo 180-8602, Japan
}

growth is suppressed by sub-MICs, and has not been investigated for all veterinary fluoroquinolones, including orbifloxacin. In this study, we examined the in vitro PAEs and PA-SMEs of orbifloxacin against Escherichia coli and Pseudomonas aeruginosa, which are representative gram-negative pathogens responsible for urinary and skin infections, respectively, in dogs, and these values were compared with those for enrofloxacin.

Two strains each of E. coli (strains 09-207 and 09-225) and $P$. aeruginosa (strains 33 and 72) were used in this study. These organisms were isolated from canine urine (E. coli) and skin (P. aeruginosa), and identified by gram stain, catalase, and oxidase tests and Api 20E kit (Bio Merieux, France). MICs of orbifloxacin and enrofloxacin were determined by the agar dilution method according to the guidelines of the Clinical and Laboratory Standards Institute [6]. E. coli ATCC 25922 and P. aeruginosa ATCC 27853 were used as quality control strains.

The PAE and PA-SME of orbifloxacin were examined in comparison with enrofloxacin, and were performed in accordance with procedures described previously [7]. Each strain was grown in the logarithmic growth phase to a concentration of approximately $5 \times 10^{6}$ colony-forming units $(\mathrm{CFU}) / \mathrm{mL}$ and was prepared for use in PAE 
experiments. One hour of exposure to orbifloxacin and enrofloxacin at twice the MIC reduced the starting inoculum by approximately $1-2 \log _{10}$ units. Growth controls with inoculum but no antibiotic were included for each experiment. Tubes were placed in a shaking water bath at $35^{\circ} \mathrm{C}$ for $1 \mathrm{~h}$. Following exposure to fluoroquinolones, bacteria were removed from the fluoroquinolones by pelleting the cells by centrifugation at appropriate conditions. The bacteria were resuspended in fresh, drug-free cationadjusted Mueller-Hinton broth (CAMHB), once again pelleted by centrifugation, and resuspended again in fresh, drug-free CAMHB. Control organisms were not exposed to either fluoroquinolone but were treated similarly. Following drug removal, the fluoroquinolone-exposed and control cultures were placed in fresh media and incubated in a water bath at $35^{\circ} \mathrm{C}$ with agitation (100 rpm). Viability counts were determined before exposure, immediately after centrifugation $(0 \mathrm{~h})$, and then hourly for $5 \mathrm{~h}$ by plate counting. A test of final colony counts was performed at $24 \mathrm{~h}$ to allow for the sufficient growth of all samples. The PAE was defined according to the formula: PAE (in hours) $=T-C$, where $T$ is the time required for viability counts of an antibiotic-exposed culture to increase by 1 $\log$ unit above counts taken immediately after dilution and $C$ is the corresponding time for the growth control, as previously described [1].

In cultures designated for PA-SME, the PA-phase $E$. coli or $P$. aeruginosa organisms were exposed to different sub-MICs $(0.1,0.2$, and 0.3 times the MIC) of orbifloxacin and enrofloxacin. One sample of PA-phase bacteria to which no drug was added served as the control. All samples and controls were incubated in a water bath at $35^{\circ} \mathrm{C}$ with agitation $(100 \mathrm{rpm})$ and the growth of all cultures was monitored by determining viable cell counts, as described above. The PA-SME was calculated using the equation: PA-SME (in hours) $=T_{P A}-C$, where $T_{P A}$ is the time required for sub-MIC-treated PA-phase organisms to grow to $1 \log$ unit and $C$ is the time required for unexposed organisms to grow to $1 \log$ unit, as previously described [1]. The PAE and PA-SME were measured in three independent experiments. The Student's $t$ test was used to determine the significant differences $(P<0.05)$ between the two groups. For each experiment, viability counts $(\log \mathrm{CFU} / \mathrm{mL})$ were plotted against time and expressed as the means of results from three separate assays. The fluctuations of bacterial numbers during experiments are shown in Figures 1, 2, 3, 4, and MICs, PAEs, and PA-SMEs are summarized in Table 1.

In this study, the average PAE of orbifloxacin for $E$. coli was not significantly different from that of enrofloxacin $(0.29 \mathrm{~h}$ vs. $0.32 \mathrm{~h}, P=0.84)$, whereas the average PAE of orbifloxacin for $P$. aeruginosa was significantly shorter than that of enrofloxacin $(0.37 \mathrm{~h}$ vs. $0.87 \mathrm{~h}, P=$ 0.022). In previous studies, van den Hoven et al. [3] reported that difloxacin had PAEs of $0.5 \mathrm{~h}$ for E. coli and $-0.4 \mathrm{~h}$ for P. aeruginosa. Spreng et al. [2] reported PAEs for marbofloxacin that ranged from 0.6 to $0.9 \mathrm{~h}$ for $E$. coli. These PAEs were induced by the exposure of the drug at twice the MIC for $1 \mathrm{~h}$, which is the same

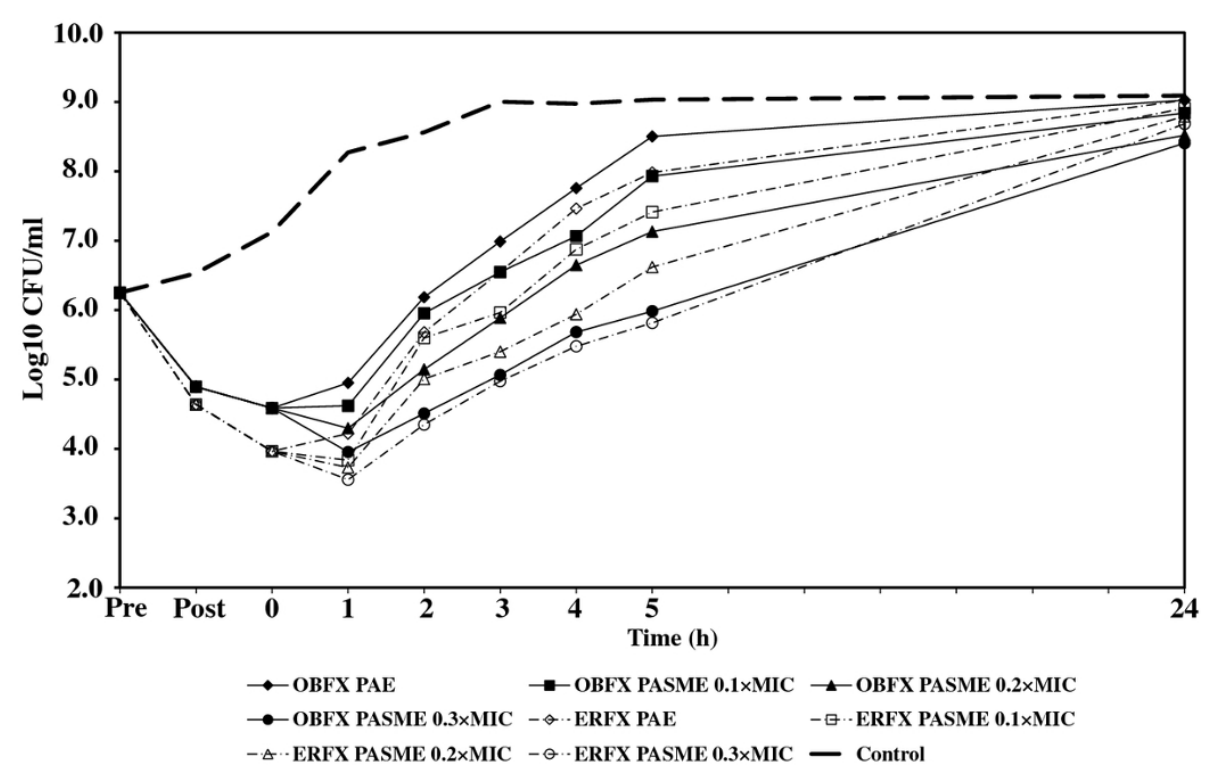

Figure 1 PAE and PA-SME of orbifloxacin and enrofloxacin for E. coli 09-207. Pre: The time of beginning exposure to the fluoroquinolone at twice the MIC. Post: The time of discontinuing exposure to the fluoroquinolone at twice the MIC 


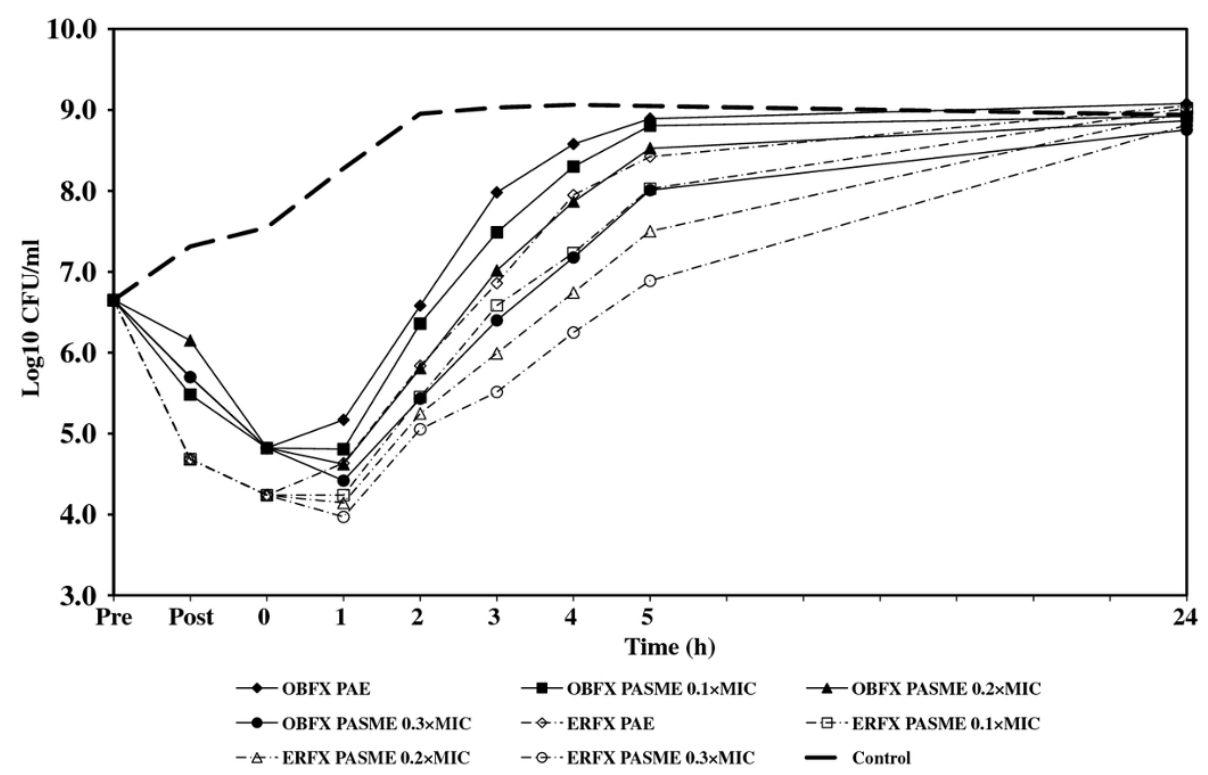

Figure 2 PAE and PA-SME of orbifloxacin and enrofloxacin for E. coli 09-225. Pre: The time of beginning exposure to the fluoroquinolone at twice the MIC. Post: The time of discontinuing exposure to the fluoroquinolone at twice the MIC.

condition as in the present study. Thus, orbifloxacin is likely to have average PAEs for E. coli and P. aeruginosa among veterinary fluoroquinolone drugs.

The PA-SMEs have been studied extensively in most antibiotics with bacterial species in human medicine [1] but not in veterinary medicine. In this study, we first determined PA-SMEs of two veterinary fluoroquinolones, orbifloxacin and enrofloxacin. Following continued exposure to orbifloxacin at $0.1,0.2$, and 0.3 times the MIC, the average PA-SMEs were observed at 0.55, 1.11, and $2.03 \mathrm{~h}$, respectively, for E. coli, and 1.04, 1.40, and $2.47 \mathrm{~h}$, respectively, for $P$. aeruginosa. On the other hand, the PA-SMEs of enrofloxacin at each concentration were $0.58,0.87$, and $1.38 \mathrm{~h}$, respectively, for $E$. coli and $1.22,1.31$, and $1.94 \mathrm{~h}$, respectively, for $P$. aeruginosa. There were no significant differences in values of PA-SMEs between orbifloxacin and enrofloxacin $(P \geq$ $0.17)$. The PA-SMEs of both drugs were significantly

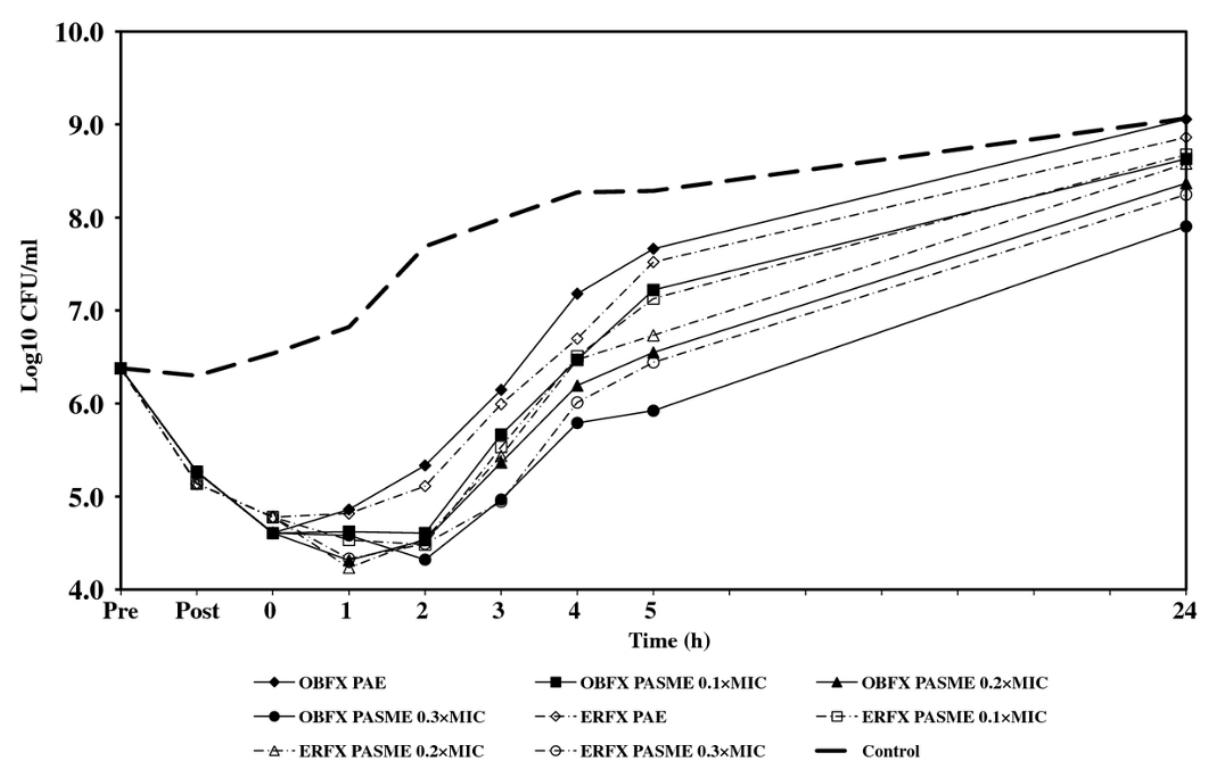

Figure 3 PAE and PA-SME of orbifloxacin and enrofloxacin for $\boldsymbol{P}$. aeruginosa 72 . Pre: The time of beginning exposure to the fluoroquinolone at twice the MIC. Post: The time of discontinuing exposure to the fluoroquinolone at twice the MIC. 


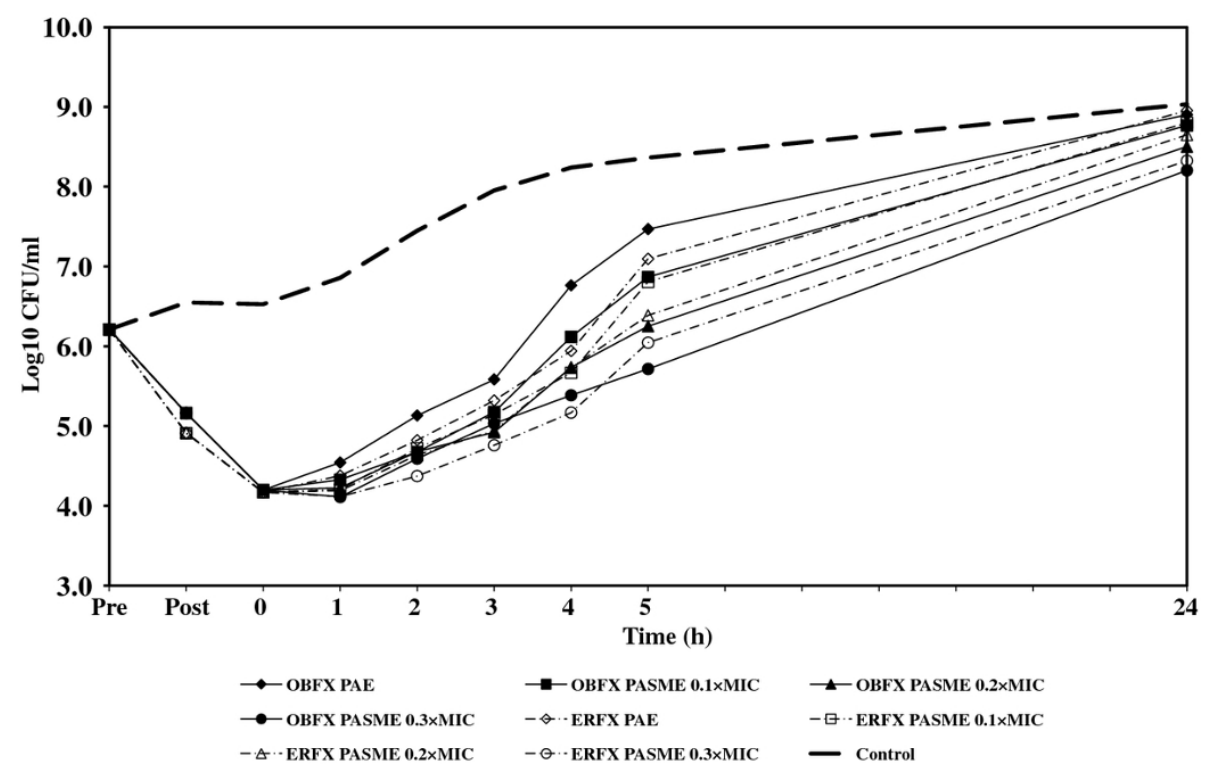

Figure 4 PAE and PA-SME of orbifloxacin and enrofloxacin for P. aeruginosa 33. Pre: The time of beginning exposure to the fluoroquinolone at twice the MIC. Post: The time of discontinuing exposure to the fluoroquinolone at twice the MIC.

longer than the corresponding PAEs $(P \leq 0.037)$, which may suggest that the PA-SME of orbifloxacin, as well as enrofloxacin, for E. coli and P. aeruginosa can be prolonged by increased sub-MICs.

The relationship between MIC and pharmacodynamic parameters, including PAE and PA-SME, is not entirely understood. This study showed that the strains with higher MICs of orbifloxacin and enrofloxacin (i.e. E. coli 09-225 and $P$. aeruginosa 33 ) consistently showed shorter PAE and PA-SME, compared with those with lower MICs (i.e. E. coli 09-207 and P. aeruginosa 72), respectively. Notably, there were significant differences in the PA-SMEs of orbifloxacin at 0.2 and 0.3 times the MIC between the two $E$. coli strains $(P \leq 0.027)$. These results suggest that MICs can affect the periods of PAE and PASME in bacteria. However, Licata et al. [7] reported that the two strains of Staphylococcus aureus, with the same MICs, showed different PAEs and PA-SMEs. Therefore, the effect of MICs on PAEs and PA-SMEs in bacteria requires further investigation.

In conclusion, our study showed that orbifloxacin has meaningful PAEs and PA-SMEs for E. coli and P. aeruginosa isolates from dogs. The exposure concentrations of orbifloxacin to induce PAE and PA-SME in this study are achievable at each infection site (i.e., urine and skin) by usual therapeutic doses $[8,9]$. For these organisms,

Table 1 PAEs and PA-SMEs of orbifloxacin and enrofloxacin for canine E.coli and $P$. aeruginosa isolates

\begin{tabular}{|c|c|c|c|c|c|c|}
\hline \multirow[t]{2}{*}{ Organism (origin) } & \multirow[t]{2}{*}{ Antibiotic } & \multirow[t]{2}{*}{ MIC ( $\mu \mathrm{g} / \mathrm{ml})$} & \multirow[t]{2}{*}{ PAE $(h)^{a, b}$} & \multicolumn{3}{|l|}{ PA SME (h) ${ }^{b}$} \\
\hline & & & & 0.1 the MIC & 0.2 the MIC & 0.3 the MIC \\
\hline \multirow[t]{2}{*}{ E. coli 09-207 } & Orbifloxacin & 0.125 & $0.53(0.12-0.93)$ & $0.80(0.54-1.27)$ & $1.61(1.31-2.00)$ & $3.04(2.68-3.70)$ \\
\hline & Enrofloxacin & 0.031 & $0.52(-0.12-0.99)$ & $0.76(0.10-1.50)$ & $1.15(0.45-2.04)$ & $1.93(1.07-2.72)$ \\
\hline \multirow[t]{2}{*}{ E. coli 09-225 } & Orbifloxacin & 2 & $0.05(-0.28-0.27)$ & $0.29(0.09-0.51)$ & $0.61(0.20-0.84)$ & $1.02(0.46-1.31)$ \\
\hline & Enrofloxacin & 0.5 & $0.13(-0.11-0.54)$ & $0.40(-0.08-0.85)$ & $0.60(0.01-1.40)$ & $0.84(0.12-1.34)$ \\
\hline \multirow[t]{2}{*}{ E. coli (mean) } & Orbifloxacin & - & 0.29 & 0.55 & 1.11 & 2.03 \\
\hline & Enrofloxacin & - & 0.32 & 0.58 & 0.87 & 1.38 \\
\hline \multirow[t]{2}{*}{ P. aeruginosa 72} & Orbifloxacin & 2 & $0.62(0.25-1.18)$ & $1.19(0.59-1.81)$ & $1.65(0.86-2.23)$ & $3.34(1.34-6.14)$ \\
\hline & Enrofloxacin & 0.5 & $1.04(0.79-1.39)$ & $1.37(1.08-1.81)$ & $1.42(1.25-1.66)$ & $2.15(1.37-2.71)$ \\
\hline \multirow[t]{2}{*}{ P. aeruginosa 33} & Orbifloxacin & 4 & $0.13(-0.18-0.50)$ & $0.88(0.55-1.78)$ & $1.14(0.54-1.90)$ & $1.60(0.64-2.27)$ \\
\hline & Enrofloxacin & 1 & $0.71(0.05-1.53)$ & $1.06(0.31-1.95)$ & $1.20(0.43-2.14)$ & $1.74(1.07-2.74)$ \\
\hline \multirow[t]{2}{*}{ P. aeruginosa (mean) } & Orbifloxacin & - & 0.37 & 1.04 & 1.40 & 2.47 \\
\hline & Enrofloxacin & - & 0.87 & 1.22 & 1.31 & 1.94 \\
\hline
\end{tabular}

${ }^{\mathrm{a}}$ Organisms exposed to two times the MIC.

${ }^{\mathrm{b}}$ Averages of three individual experiments; ranges in parentheses. 
however, other important factors affecting antimicrobial potency (e.g. bactericidal effect [10] and postantibiotic leukocyte enhancement [11]) remain to be clarified. To additionally validate regimen of veterinary fluoroquinolones including orbifloxacin, further studies would be needed.

\section{Authors' contributions}

$\mathrm{KH}$ and TS carried out all experiments and equally contributed to this study. $\mathrm{KH}$ was involved in the study design. $\mathrm{KH}, \mathrm{YK}$, and $\Pi \mathrm{T}$ was preparation of the manuscript. KH drafted the manuscript. All authors read and approved the final manuscript.

\section{Competing interests}

This study was supported by a grant from DS Pharma Animal Health Co., Ltd., Japan. The sponsor of the study had no role in the study design, conduct of the study, data collection, data interpretation or preparing of the manuscript.

Received: 4 October 2011 Accepted: 20 March 2012

Published: 20 March 2012

\section{References}

1. Odenhold I: Pharmacodynamic effects of subinhibitory antibiotic concentrations. Int J Antimicrob Agents 2001, 17:1-8.

2. Spreng M, Deleforge J, Thormas V, Boisramé B, Drugeon H: Antibacterial activity of marbofloxacin. A new fluoroquinolone for veterinary use against canine and feline isolates. J Vet Pharmacol Ther 1995, 18:284-289.

3. Van den Hoven R, Wagenaar JA, Walker RD: In vitro activity of difloxacin against canine bacterial isolates. J Vet Diagn Invest 2000, 12:218-223.

4. Carbone M, Pennisi MG, Masucci M, De Sarro A, Giannone M, Fera MT: Activity and postantibiotic effect of marbofloxacin, enrofloxacin, difloxacin and ciprofloxacin against feline Bordetella bronchiseptic isolates. Vet Microbiol 2001, 81:79-84.

5. Fera MT, Losi E, Pennisi MG, Masucci M, Giannone M, Maugeri TL, Carbone M: Potency and postantibiotic effect of four fluoroquinolones against feline Pasteurella multocid isolates. Vet Rec 2002, 151:180-181.

6. Clinical and Laboratory Standards Institute: Performance Standards for Antimicrobial Disk and Dilution Susceptibility Tests for Bacteria Isolated From Animals; Approved Standard - Third Edition. CLSI document M31-A3 Wayne, PA; 2008

7. Licata L, Smith CE, Goldschmidt RM, Barrett JF, Frosco M: Comparison of the postantibiotic and postantibiotic sub-MIC effects of levofloxacin and ciprofloxacin on Staphylococcus aureu and Streptococcus pneumonia. Antimicrob Agents Chemother 1997, 41:950-955.

8. Matsumoto S, Takahashi M, Yoshida M, Komatsu T, Kitadai Y, Horii Y, Katae $\mathrm{H}$ : Absorption, distribution and excretion of orbifloxacin in dogs and cats. Jan J Vet Med Assoc 1997, 50:470-474, in Japanese with English summary.

9. Kay-Mugford PA, Weingarten AJ, Ngoh M, Zolynas R, White A, Katz T, Simmons R, Varma KJ: Determination of plasma and skin concentrations of orbifloxacin in dogs with clinically normal skin and dogs with pyoderma. Vet Ther 2002, 3:402-408

10. Ganière JP, Médaille C, Etoré F: In vitro antimicrobial activity of orbifloxacin against Staphylococcus intermediu isolates from canine skin and ear infections. Res Vet Sci 2004, 77:67-71.

11. Novelli A, Mazzei T, Fallani S, Cassetta Ml, Conti S: In vitro postantibiotic effect and postantibiotic leukocyte enhancement of tobramycin. J Chemother 1995, 7:355-362.

doi:10.1186/1751-0147-54-16

Cite this article as: Harada et al.: Post-antibiotic effect of orbifloxacin against Escherichia coli and Pseudomonas aeruginosa isolates from dogs. Acta Veterinaria Scandinavica 2012 54:16.

\section{Submit your next manuscript to BioMed Central and take full advantage of:}

- Convenient online submission

- Thorough peer review

- No space constraints or color figure charges

- Immediate publication on acceptance

- Inclusion in PubMed, CAS, Scopus and Google Scholar

- Research which is freely available for redistribution

Submit your manuscript at www.biomedcentral.com/submit
Ciomed Central 